\title{
Double unilateral fenestration of the anterior cerebral artery in the pre-communicating segment: a report of a unique case
}

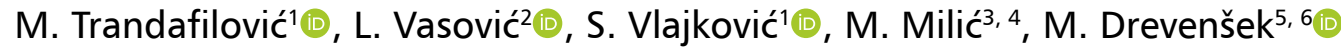 \\ ${ }^{1}$ Department of Anatomy, Faculty of Medicine, University of Niš, Serbia \\ ${ }^{2}$ Faculty of Medicine, University of Niš, Serbia \\ ${ }^{3}$ Department of Forensic Medicine, Faculty of Medicine, University of Niš, Serbia \\ ${ }^{4}$ Department of Forensic Medicine in Niš, Serbia \\ ${ }^{5}$ Department of Orthodontics, Faculty of Medicine, University of Ljubljana, Slovenia \\ ${ }^{6}$ Department of Orthodontics, Ljubljana University Medical Centre, Ljubljana, Slovenia
}

[Received: 19 July 2021; Accepted: 6 August 2021; Early publication date: 15 September 2021]

\begin{abstract}
A unique example of two fenestrations of the pre-communicating (A1) segment of the right anterior cerebral artery (ACA) in a 78-year-old woman was a special case among 388 cadaveric specimens. It was found by a retrospective review of the personal data obtained during graduate and undergraduate studies at the Faculty of Medicine.

Two unequal fenestrations of the right A1 segment were associated with the presence of a pseudo anterior communicating artery, hypoplasia of the right posterior communicating artery and the left superior cerebellar artery, absence of the right anterior inferior cerebellar artery, and ectasia of the basilar artery; however, the cerebral arteries were without aneurysm(s) or other pathology. The reason could be the almost equal ACA diameter on both sides. (Folia Morphol 2022; 81, 4: 1058-1061)
\end{abstract}

Key words: anterior cerebral artery, fenestration, brain base, associated arterial variations

\section{INTRODUCTION}

The anterior cerebral artery (ACA), as one of the two terminal branches of the cerebral part of the corresponding internal carotid artery (ICA), courses through pre- (A1) and post-communicating (A2) segments. It runs anteromedially and anastomoses with the opposite ACA via the anterior communicating artery (ACOA), and continues to pass between the cerebral hemispheres over the genu towards the splenium of the corpus callosum; it branches into (sub)cortical arteries as well as branches for basal nuclei [3].
There were a wide range of ACA variations, such as hypoplasia $[4,18]$, variable origin and/or course $[9,13]$, variable branching $[6,14]$, and/or abnormalities, such as a simplification of the paired ACAs into an azygos trunk [4, 11, 14], aplasia [8], and persistence of the primitive (plexiform) shape of the A1 subpart [12], along the whole $A 1$ part [1], or as a button-hole defect or a fenestration of the artery $[4,7,8,14]$.

Fenestrations of single cerebral arteries of the carotid (CS) and vertebrobasilar (VBS) systems (ICA, ACA, middle/posterior cerebral arteries, anterior/ /posterior communicating arteries, vertebral arteries,

Address for correspondence: M. Trandafilović, MD, Assistant Professor, Faculty of Medicine, Department of Anatomy, 81 Dr Zoran Đinđić Blvd., 18000 Niš, Serbia, tel: +381 184570 029, fax: +381 184238 770, e-mail: milena.trandafilovic@medfak.ni.ac.rs

This article is available in open access under Creative Common Attribution-Non-Commercial-No Derivatives 4.0 International (CC BY-NC-ND 4.0) license, allowing to download articles and share them with others as long as they credit the authors and the publisher, but without permission to change them in any way or use them commercially. 

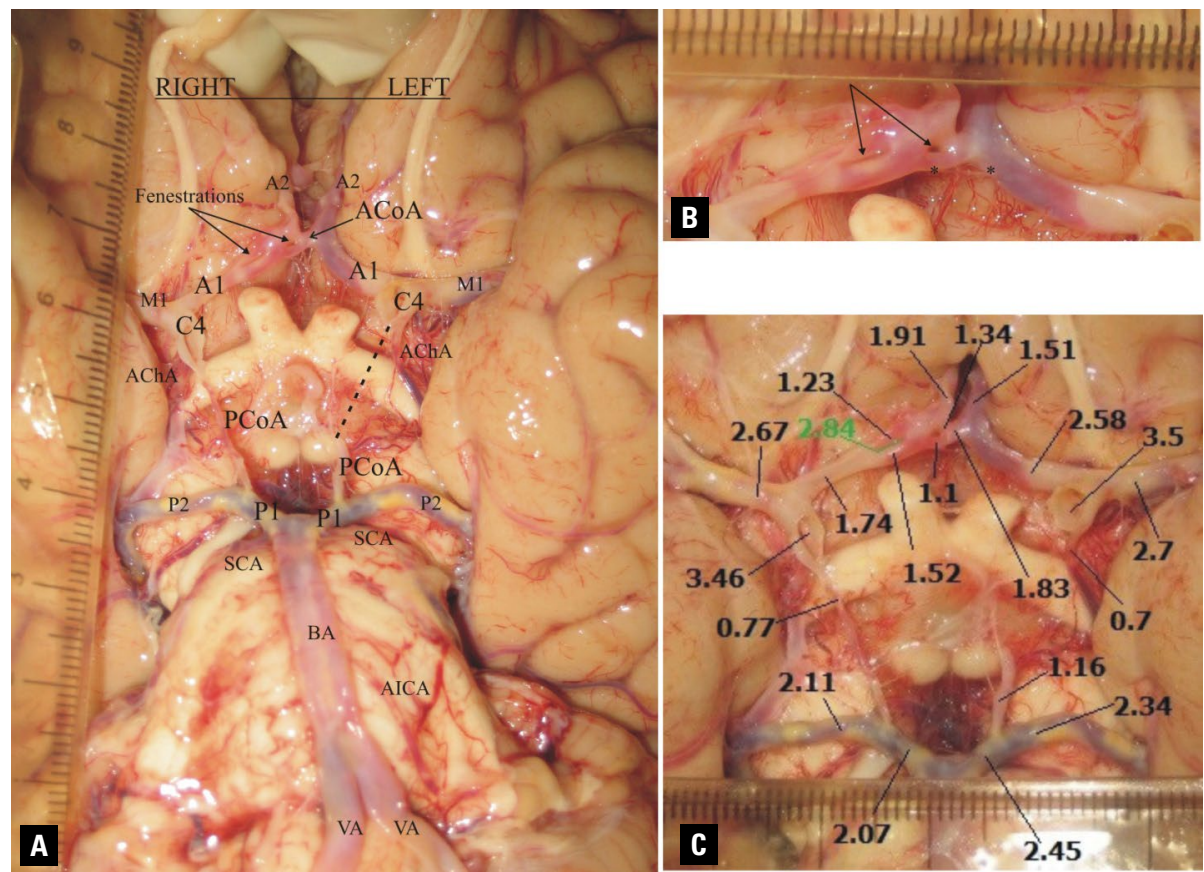

Figure 1. Case of double unilateral fenestration of the anterior cerebral artery (ACA); A. View of the cerebral angioarchitecture on the brain base; $B$. Focus on the right $A 1$ part and anterior communicating artery ( $\left.A C_{0} A\right)$. Two arrows indicated two fenestrations on the right $A 1$ part; two stars limited the origin and length of so-called pseudo ACoA; C. Values of the outer diameter $(\mathrm{mm})$ of some main arteries on the brain base; the length of larger fenestration in the right $A 1$ part is marked by green colour; $A 2$ - post-communicating part of ACA; $A 1$ - pre-communicating part of ACA; $M 1$ - sphenoidal part of the middle cerebral artery; $\mathrm{C4}$ - cerebral part of the internal carotid artery (ICA); $\mathrm{AChA}$ anterior choroidal artery; $\mathrm{PCoA}$ - posterior communicating artery (broken line indicated the left PCoA to the ICA origin); $\mathrm{P} 1$ - pre-communicating part of the posterior cerebral artery (PCA); $\mathrm{P} 2$ - post-communicating part of PCA; SCA — superior cerebellar artery; $B A$ - basilar artery; AICA — anterior inferior cerebellar artery; VA — vertebral artery.

basilar artery, and posterior inferior cerebellar arteries) in single or multiple appearances $[2,4,14]$, or associated with each other [15] have been described.

The theories about the origin and pathoanatomical significance of the cerebral artery fenestration cited in Scranton et al. [15] are still controversial. We will present two fenestrations of the right $\mathrm{A} 1$ segment without aneurysm(s) or other pathology in a cadaver in the eighth decade of age.

\section{CASE REPORT}

A unique case of double fenestration of the unilateral $A 1$ segment was found in a retrospective review of the data of 388 adult cadavers of various ages, genders and causes of death autopsied at the Department of Forensic Medicine in Niš.

The double fenestration in the right $A 1$ segment on the brain base was found in a 78-year-old female cadaver, autopsied due to an incidental polytrauma on the street.

The cerebral angioarchitecture of the brain base was recorded on film and schematised in a workbook. At first, the cerebral arteries were observed macroscopically and with a magnifying glass, and the outer diameter (OD) of each artery was calculated from the digital images, using the ImageJ processing programme (http://rsb.info.nih.gov/ij/index.html).

The vessel's OD of $\leq 1.0 \mathrm{~mm}$ in the $\mathrm{CS}$, as cited in Vasović et al. [18], was the threshold for the explanation of its size as a hypoplasia, while data from another article [17] were used for the explanation of the eventual (dolicho)ectasia of an artery in the VBS.

Figure $1 A, C$ shows the cerebral angioarchitecture of the brain base as follows: the cerebral arterial circle (CAC) was of a nonagon shape, and it was formed by the subparts of the cerebral segment (C4) of paired ICAs; paired A1 segments connected by the $A C o A$; as vascular components of the anterior CAC segment, paired posterior communicating arteries (PCoAs) and pre-communicating (P1) segments of the posterior cerebral arteries (PCAs); and as vascular components of the posterior CAC segment. The ODs of all the CAC vascular components were in the normal range, except the right $\mathrm{PCoA}$, which was hypoplastic. 
Two fenestrations of the right $A 1$ segment causing a bifenestrated $A 1$ segment were visible; one was located in the middle third of the A1 segment, of an ellipsoid shape (2.84 $\mathrm{mm}$ in length; see Fig. $1 \mathrm{~A}-\mathrm{C}$ ), and the second was of a semilunar form $(1.1 \mathrm{~mm}$ in length) and was positioned at the A1 end (immediately before its junction with the ACOA).

The OD of the ACoA was $1.83 \mathrm{~mm}$; a caudally positioned threadlike vessel transversely oriented, as a "pseudo ACoA", connected the two A1 parts below and laterally from the level of the ACA-ACoA junction (stars in Fig. 1B). This was a vascular source of two suprahiasmatic branches.

The right $\mathrm{PCOA}$ was hypoplastic $(\mathrm{OD}=0.77 \mathrm{~mm})$; the left PCOA was of a normal OD $(1.16 \mathrm{~mm})$, but it was artificially ruptured.

The basilar artery (BA) was ectatic $(4.89 \mathrm{~mm})$; however, its branches - the left superior cerebellar (SCA) and the anterior inferior cerebellar (AICA) arteries were hypoplastic, while the right SCA was of a normal $\mathrm{OD}$, and the right AICA was absent. Both vertebral arteries (VAs) had normal ODs (3.84 mm on the right; $3.31 \mathrm{~mm}$ on the left); their convergent junction had a slight dislocation on the left (immediately above the left pyramid on the ventral side of the myelencephalon).

Insular atheromatous plaques were present in the main arteries of the CS and VBS.

\section{Ethical considerations}

The protocol was approved by the Council on Graduate Study and Research Ethics Committee (No. 01-9068-4) of our Faculty of Medicine. There were no financial or commercial gains and the authors declare that they have no conflicts of interest.

\section{DISCUSSION}

In general, arterial "fenestration" is described if the (cerebral) artery is doubled in one part of its trunk into two independent channels with their own tunica intima and tunica media and, sometimes with a tunica adventitia, and after the appropriate course these channels reform the original artery $[2,4,15]$.

The first available description of the $A 1$ fenestration was found in an article by Fawcett and Blachford [5], giving an incidence of $0.14 \%$. Since then there have been many articles including this vascular abnormality, such as case reports $[1,7,8]$, or review [14] or retrospective $[2,4,10]$ studies.

Fenestration of the $A 1$ segment may occur, as cited in Iwabuchi et al. [7], due to the absence of the fu- sion of the primitive plexiform anastomosis between the ACA and the primitive olfactory artery, normally presented at the 18-43 mm embryonic stage. Consequently, developmental failure of the ACA may increase blood flow in one of the $A 1$ segments because of possible A1 hypoplasia [7] or A1 aplasia [8, 19] on the opposite side, and results in increased haemodynamic stress and defects in the tunica media of the ipsilateral A1 bifurcation or fenestration, and finally in the formation of an aneurysm [4].

Our case was similar to a case published by Yoshida et al. [19]; however, although the previous authors noted a "tandem" of A1 and ACOA fenestrations in a 50-year-old man, associated with an $\mathrm{A} 1$ aneurysm and contralateral A1 aplasia, we are of the opinion that the ACoA could not have existed, due to the opposite ACA aplasia.

The association of vascular variations, such as the presence of a pseudo ACoA, hypoplasia of the right PCoA and left SCA, the absence of the right AICA, and BA ectasia with (two) fenestrations of the right $A 1$ segment in the presented case, is a unique case in available literature. Badagabettu et al. [1] described a hypoplastic plexiform right $A 1$ segment followed by an absence of the ACoA which was replaced by an azygos trunk. Kachhara et al. [8] described an opposite A1 aplasia, while Kobayashi et al. [11] and Uchino et al. [16] presented a contralateral A1 hypoplasia and azygos trunk.

Some findings of ACA fenestrations in the literature indicated controversies about their pathophysiological and clinical significance. They are as follows: 1) Kim et al. [10] performed a retrospective review of 843 conventional cerebral angiograms and 1,787 cranial magnetic resonance angiograms (MRAs) in South Korea, and found 14 cases of A1 fenestrations, with 3 of the 14 cases being associated with aneurysms; simultaneously, an ACA aneurysm was evidenced in only 1 case; 2 ) Uchino et al. [16] found 9/891 cases of A1 fenestrations without any pathological signs in the MRAs of patients in Japan; 3) A proof of the relationships between ACA fenestrations and aneurysms can be found in a paper published by Cooke et al. [2]; they discovered cerebral arterial fenestrations in 228/10,927 patients (2.1\%) in the United States using 20-year-old digital subtraction angiograms from their clinical archives; at the same time, they found ACA fenestrations in 43 cases ( $43 / 228$ or $18.9 \%)$, and of them 26 cases ( $26 / 43$ or $61.9 \%$ ) were associated with aneurysms; 4) Although there 
have been case reports about different aneurysm locations independent of a finding of ACA fenestration $[11,12]$, Yoshida et al. [19] noted that up to the year 2012 a total of 21 cases (including their own case) of aneurysms arising from the $A 1$ fenestration had been recorded; and 5) Clinically, a small fenestration of ACA, such as the second one in the presented case, could be mistaken for an ACoA aneurysm on an MRA [14].

\section{CONCLUSIONS}

Although it is very well known that the ACoA and the $B A$, as unpaired arteries on the median line of the brain base, characterise the appearance of multiple fenestrations, the presented case of a double unilateral ACA fenestration is proof of the possibility of a break in the vessel's continuity out of the median line during its simplification caused by persistent arterial precursors, such as the ACA in this case.

Fenestrations of the cerebral arteries of the CS can be associated with vascular variations of the same and the VB systems.

Fenestration(s) of the A1 segment are not "weak points" for aneurysm development, especially if the ACAs' calibre does not vary on different sides.

\section{Acknowledgements}

This study was funded by the Ministry of Science and Technological Development of the Republic of Serbia (Grant Nos. 451-03-9/2021-14/200113; 41018, 175092), an internal project of the Faculty of Medicine, University of Niš (no. 38/20), and the Slovenian Research Agency (P3-0293 research programme).

\section{Conflict of interest: None declared}

\section{REFERENCES}

1. Badagabettu SN, Guru A, Shetty SD, et al. Hypoplastic plexiform right anterior cerebral artery and absence of anterior communicating artery: a case report. Forensic Med Anat Res. 2013; 3(1): 47-49, doi: 10.4236/ fmar.2013.13009.

2. Cooke DL, Stout CE, Kim WT, et al. Cerebral arterial fenestrations. Interv Neuroradiol. 2014; 20(3): 261-274, doi: 10.15274/INR-2014-10027, indexed in Pubmed: 24976087.

3. Dauber W. Pocket atlas of human anatomy, 5th rev. edn. Thieme, Stuttgart-New York 2007.

4. Dimmick SJ, Faulder KC. Normal variants of the cerebral circulation at multidetector CT angiography. Radiographics. 2009; 29(4): 1027-1043, doi: 10.1148/rg.294085730, indexed in Pubmed: 19605654.

5. Fawcett $\mathrm{E}$, Blachford JV. The circle of Willis: an examination of 700 specimens. J Anat Phys. 1905; 40(Pt 1): 63, indexed in Pubmed: 1287340.
6. Hosur B, Singh P, Kumar A, et al. Persistent primitive olfactory artery: a hairpin easy to miss! BMJ Case Rep. 2018; 11(1), doi: 10.1136/bcr-2018-227782, indexed in Pubmed: 30567897.

7. Iwabuchi N, Saito A, Fujimoto K, et al. Unruptured saccular aneurysm arising from the fenestrated $A 1$ segment of the anterior cerebral artery: report of 2 cases. Case Rep Neurol. 2018; 10(2): 140-149, doi: 10.1159/000488478, indexed in Pubmed: 30022945.

8. Kachhara R, Nair S, Gupta AK. Fenestration of the proximal anterior cerebral artery (A1) with aneurysm manifesting as subarachnoid hemorrhage: case report. Neurol Med Chir (Tokyo). 1998; 38(7): 409-412, doi: 10.2176/nmc.38.409, indexed in Pubmed: 9745246.

9. Katoh M, Kamiyama H, Makino K, et al. Infra-optic course of the anterior cerebral artery. J Clin Neurosci. 1999; 6(3): 252-255, doi: 10.1016/s0967-5868(99)90516-7, indexed in Pubmed: 18639164.

10. Kim TH, Lee HK, Rhee JJ, et al. The incidence and clinical significance of fenestrations in the hizontal segment of the anteri cerebral artery detected by conventional angiography and magnetic resonance angiography. J Korean Neurosurg Soc. 2006; 40(2): 74-78.

11. Kobayashi S, Tatsuo Y, Sugita $Y$, et al. Azygos anterior cerebral artery aneurysm with fenestration of the anterior cerebral artery. Kurume Med J. 1986; 33(3): 149-153, doi: 0.2739/kurumemedj.33.149, indexed in Pubmed: 3599873.

12. Koh JS, Lee SH, Bang JS, et al. Three-dimensional angiographic demonstration of plexiform fenestrations of the proximal anterior cerebral artery associated with a ruptured aneurysm. J Korean Neurosurg Soc. 2008; 44(5): 338-340, doi: 10.3340/ jkns.2008.44.5.338, indexed in Pubmed: 19119472.

13. Milenković Z. Anastomosis between internal carotid artery and anterior cerebral artery with other anomalies of the circle of Willis in a fetal brain. J Neurosurg. 1981; 55(5): 701-703, doi: 10.3171/jns.1981.55.5.0701, indexed in Pubmed: 7310491.

14. Okahara $M$, Kiyosue $H$, Mori $H$, et al. Anatomic variations of the cerebral arteries and their embryology: a pictorial review. Eur Radiol. 2002; 12(10): 2548-2561, doi: 10.1007/ s00330-001-1286-x, indexed in Pubmed: 12271398.

15. Scranton RA, Jenson AV, Suresh R, et al. Multiple anterior and posterior circulation intracranial fenestrations presenting with aneurysmal subarachnoid hemorrhage. Cureus. 2021; 13(1): e12667, doi: 10.7759/cureus.12667, indexed in Pubmed: 33604207.

16. Uchino A, Nomiyama K, Takase $Y$, et al. Anterior cerebral artery variations detected by MR angiography. Neuroradiology. 2006; 48(9): 647-652, doi: 10.1007/s00234-0060110-3, indexed in Pubmed: 16786350.

17. Vasović L, Trandafilović M, Jovanović l, et al. Vertebral and/ or basilar dolichoectasia in human adult cadavers. Acta Neurochir (Wien). 2012; 154(8): 1477-1488, doi: 10.1007/ s00701-012-1400-7, indexed in Pubmed: 22664729.

18. Vasović L, Trandafilović M, Jovanovićl, et al. Morphology of the cerebral arterial circle in the prenatal and postnatal period of Serbian population. Childs Nerv Syst. 2013; 29(12): 2249-2261, doi: 10.1007/s00381-013-2151-3, indexed in Pubmed: 23702737.

19. Yoshida M, Ezura M, Sasaki K, et al. Endovascular repair of ruptured aneurysm arising from fenestration of the horizontal segment of the anterior cerebral artery: case report. Neurol Med Chir (Tokyo). 2012; 52(12): 924-927, doi: 10.2176/nmc.52.924, indexed in Pubmed: 23269052. 